\title{
Synthesis and Importance of Bulky Aromatic Cap of Novel SAHA Analogs for HDAC Inhibition and Anticancer Activity
}

\author{
Pusoon Chun, Won Hee Kim, Jungsu Kim, Jin-Ah Kang, Hye Jin Lee, Ji Young Park, \\ Mee Young Ahn, Hyung Sik Kim, and Hyung Ryong Moon* \\ College of Pharmacy and Research Institute for Drug Development, Pusan National University, Busan 609-735, Korea \\ ${ }^{*}$ E-mail: mhr108@pusan.ac.kr \\ Received March 15, 2011, Accepted April 12, 2011
}

\begin{abstract}
On the basis of potent HDAC-inhibitory activity and anticancer activity of SAHA, novel SAHA derivatives 3a-d and 7 with a bulky cap such as $p$-dimethylaminophenyl, 4-phenylaminophenyl, 4-phenyloxyphenyl, $9 H$ fluorenyl or naphthalenyl ring were synthesized starting from the corresponding aryl amines or naphthalenyl acetic acid using an EDC-mediated amide coupling reaction in the presence of HOBt followed by a nucleophilic addition-elimination reaction with hydroxylamine. Compounds $\mathbf{3 b}, \mathbf{3 c}$ and $\mathbf{3 d}$ showed more potent inhibitory activity on total HDACs (14 27-fold), HDAC1 (8 15-fold), HDAC2 (1.3 25-fold) and HDAC7 (1 3-fold) and more potent anticancer activity (2 22-fold) against MCF-7, MDA-MB-231, MCF-7/ Dox, MCF-7/Tam, SK-OV-3, LNCaP and PC3 human cancer cell lines than SAHA.
\end{abstract}

Key Words : HDAC inhibition, Anticancer activity, SAHA, Bulky cap

\section{Introduction}

Nucleosomes are made up of DNA wrapped around an octamer of histones. Post-transcriptional modifications of the positively charged $N$-terminal regions of the histones, including acetylation, methylation and phosphorylation, change the chromatin structure and regulate gene transcriptions. ${ }^{1,2}$ Histone deacetylases (HDACs) and histone acetyltransferases (HATs) are involved in epigenetic regulation of genes through modifications of histone tails of $\mathrm{H} 3$ and $\mathrm{H} 4 .^{3}$ HDAC enzymes have been noticed as a very promising target for anticancer agents, due to having ability for an epigenetic modification. Recent studies have shown that inhibition of HDACs silences the proliferation of tumor cells by inducing cell-cycle arrest, terminal differentiation, and/or apoptosis. $^{4,5}$
The HDACs can be divided into two families: (1) the $\mathrm{Zn}^{2+}$ dependent HDAC family composed of class I (HDACs 1, 2, 3 and 8), class II a/b (HDACs 4, 5, 6, 7, 9 and 10), and class IV (HDAC 11) and (2) $\mathrm{Zn}^{2+}$ independent NAD ${ }^{+}$-dependent class III SIRT enzymes. ${ }^{6}$ The class I HDACs, the true histone deacetylases are localized in the nucleus of cells. The class II a/b deacetylases have both histone and nonhistone proteins as substrates and are primarily localized in the cytoplasm.

Many synthetic and naturally occurring HDAC inhibitors such as suberoylanilide hydroxamic acid (SAHA, vorinostat, Zolinza $\left.^{\circledR}\right),{ }^{7}$ apicidin, ${ }^{8}$ trichostatin $\mathrm{A},{ }^{9}$ psammaplin $\mathrm{A}^{10}$ and trapoxin $\mathrm{B}^{11}$ have been reported (Fig. 1). Hydroxamic acidtype HDAC inhibitors including SAHA reduced the tumor burden of breast, melanoma, prostate, ovarian, colon, lung and cervical cancers. ${ }^{12,13}$ SAHA is a class I/II synthetic<smiles>CCC(C)[C@H](N)C(=O)N1CCCC[C@H]1C(N)=O</smiles>

Figure 1. Synthetic and naturally occurring HDAC inhibitors. 
inhibitor, and the hydroxamic acid is the crucial moiety to obtain high inhibitory activity, since modifications or substitutions at this site reduce their anti-HDAC effect. ${ }^{14}$

SAHA reacts with and blocks the catalytic site of HDAC enzymes. SAHA can cause growth arrest and death of a broad variety of transformed cells both in vitro and in tumorbearing animals at concentrations not toxic to normal cells. ${ }^{12,15,16}$ SAHA has many protein targets whose structure and function are altered by acetylation, including chromatinassociated histones, nonhistone gene transcription factors and proteins involved in regulation of cell proliferation, migration and death. A new drug application was approved by the US Food and Drug Administration for SAHA for treatment of cutaneous T-cell lymphoma.

The common pharmacopore of the inhibitors for class I/II HDAC enzymes is composed of three parts: (1) a hydrophobic region that binds a cap moiety of inhibitors, (2) an enzyme inhibition region chelating with a zinc cation near the bottom of the catalytic pocket, and (3) a linker region connecting the hydrophobic region with the enzyme inhibition region. ${ }^{17}$

According to SAR studies previously reported, HDAC inhibitors with a four- to six-atom linker and the functionality of hydroxamic acid that can bind the enzyme inhibition region showed very good activity. ${ }^{17}$ Thus, we investigated the cap effect of HDAC inhibitors on the inhibitory activities against HDACs and several human cancer cell lines, without any structural changes of the hexamethylene linker and the functionality of hydroxamic acid of SAHA. In addition, the effect of transposed amide functionality was examined on the inhibitory activities against HDACs and anticancer activity.

As part of our continuous efforts searching for novel potent HDAC inhibitors, it was interesting to synthesize
SAHA analogues with a bulky cap such as 4-phenoxyphenyl, 4-dimethylaminophenyl, and 4-phenylaminophenyl rings, instead of the phenyl ring of SAHA, and a transposed amide analog with a naphthalenyl ring at the cap moiety. Herein, we wish to report the synthesis of novel SAHA derivatives possessing a bulky cap, and their inhibitory activities against HDAC enzymes and several human cancer cell lines.

\section{Results and Discussion}

Chemistry. Common structural feature of all the final compounds 3a-c (Scheme 1) is that they bear a hexamethylene unit as their linkers and hydroxamic acid to bind to zinc cation existing at the active site of HDAC. A strategy for the synthesis of SAHA derivatives is outlined in Scheme 1. Reaction of suberic acid monomethyl ester with aromatic amines such as $N^{1}, N^{1}$-dimethylbenzene-1,4-diamine, 4phenoxyaniline, and $N$-phenyl-p-phenylenediamine in the presence of EDC ( $N$-ethyl- $N$-(3-dimethylaminopropyl)carbodiimide) hydrochloride and HOBt (1-hydroxybenzotriazole $)^{18,19}$ gave the corresponding amides, respectively, in $49-62 \%$ yields. Although the long reaction time of 5-10 days was needed, treatment of methyl esters 2a-c with $50 \%$ hydroxylamine aqueous solution afforded SAHA analogs 3a-c in 57-67\% yields.

Compound 7 in which the amide functionality of the SAHA structure was transposed and in which the phenyl ring of SAHA was replaced by a naphthalenyl ring was synthesized to evaluate an effect of the reversed amide functionality on HDAC inhibition and anticancer activity (Scheme 2). For the synthesis of compound 7 with the reversed amide functionality, commercially available 1-

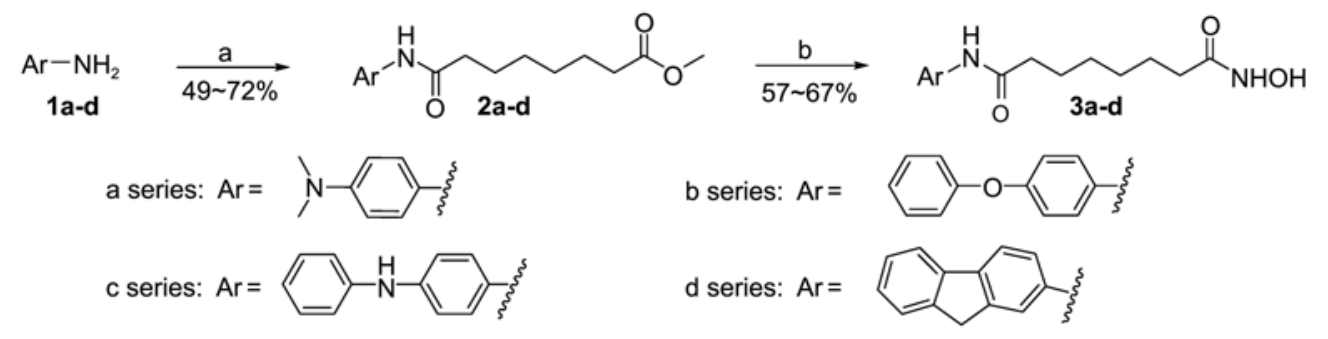

Reagents and conditions:

(a) suberic acid monomethyl ester, EDC, $\mathrm{HOBt}, \mathrm{CH}_{2} \mathrm{Cl}_{2}, \mathrm{rt}, 2 \sim 4 \mathrm{~d}$; (b) $50 \% \mathrm{NH}_{2} \mathrm{OH}$ aqueous solution, $\mathrm{MeOH}, \mathrm{rt}, 5-10 \mathrm{~d}$.

Scheme 1. Synthesis of the target compounds $\mathbf{3 a - 3 d}$.
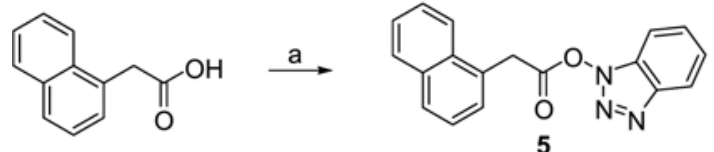

Reagents and conditions: (a) HOBt, EDC, 1,4-dioxane, rt, $2 \mathrm{~h}$; (b) methyl 6-aminohexanoate hydrochloride, $\mathrm{N}$-methyl morpholine, 1,4-dioxane, rt, $2 \mathrm{~d}$; (c) $50 \% \mathrm{NH}_{2} \mathrm{OH}$ aqueous solution, $\mathrm{MeOH}, \mathrm{rt}, 2 \mathrm{~d}$.

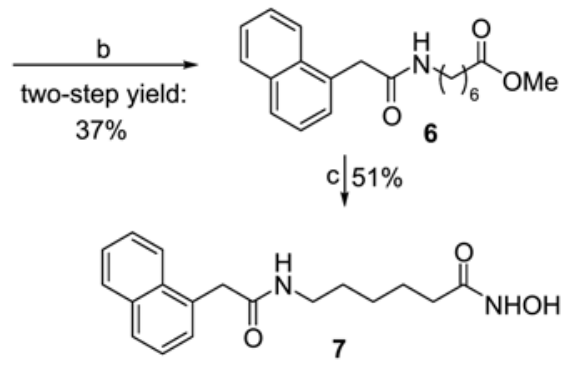

Scheme 2. Synthesis of the target compound 7. 
naphthaleneacetic acid was used. First, reaction of 1naphthaleneacetic acid with HOBt in the presence of EDC gave 1-naphthaleneacetic acid-HOBt ester adduct 5, which was coupled with methyl 6-aminohexanoate in the presence of $\mathrm{N}$-methyl morpholine as a tertiary amine catalyst to give the reversed amide compound $\mathbf{6}$ in $37 \%$ yield from naphthaleneacetic acid. Treatment of compound 6 with $50 \%$ hydroxylamine aqueous solution converted methyl ester into hydroxamic acid to afford compound 7 in a moderate yield. It should be noted that the number of the linker atoms between naphthalenyl ring and hydroxamic acid in compound 7 is same as that between phenyl ring and hydroxamic acid in SAHA.

HDAC Enzymes Inhibition. The effects of the final compounds 3a-c and 7 on total HDACs, HDAC1, HDAC2, HDAC3, HDAC4, and HDAC7 enzyme activity in nuclear protein isolated from the compound-treated SK-OV-3 cells were examined. As shown in Table 1, compounds $\mathbf{3 b}$ and $\mathbf{3 c}$ inhibited the total HDAC enzymes, HDAC1, HDAC2, and HDAC7 very potently and the $\mathrm{IC}_{50}$ values were lower than those of SAHA used as a positive control. In case of HDAC3 and $\mathrm{HDAC} 4, \mathrm{IC}_{50}$ values of $\mathbf{3 b}$ and $\mathbf{3} \mathbf{c}$ were similar to those of SAHA. In total HDACs and HDAC1, HDAC enzymeinhibitory activity of compound $\mathbf{3 a}$ was similar to or a little higher (2 6 fold times) than that of SAHA, but in the other HDACs examined, the inhibitory activities were similar to or lower than that of SAHA. A common characteristic of $\mathbf{3 b}$ and $\mathbf{3 c}$ is to markedly inhibit both HDAC1 and HDAC7 enzymes as well as the total HDAC enzymes, compared with SAHA. Compounds $\mathbf{3 b}$ and $\mathbf{3 c}$ inhibited the total HDACs activity in more than 13-fold and 17-fold, respectively and HDAC1 activity in more than 7-fold and 10-fold, respec- tively, compared with SAHA. However, compound 7 with reversed amide functionality exhibited lower inhibitory activity than SAHA against HDACs examined. Our findings show that $\mathbf{3 b}$ and $\mathbf{3 c}$ are much more significant HDACs inhibitors than SAHA on both total HDACs enzymes and each HDACs enzyme. The superior inhibition to SAHA might result from the bulkiness of their cap moieties. Therefore, compound 3d with a fluorenyl group as a bulky cap was synthesized and its HDACs inhibitions were examined. As expected, 3d exhibited the most potent inhibitory effect on total HDAC enzymes, HDAC1 and HDAC2 (27-, 15and 25-fold potent, respectively, compared to SAHA) and a similar inhibitory effect on HDAC3 to compounds $\mathbf{3 b}$ and 3c. These results imply that the cap size is very closely related to HDAC inhibitory activity.

Anticancer Activity. The therapeutic efficacy of HDAC inhibitors was tested in a variety of human cancer cell lines including breast cancer cell lines (MCF-7, MDA-MB-231, MCF-7/Dox, and MCF-7/Tam), ovary cancer cell line (SKOV-3) and prostate cancer cell lines (LNCaP, DU145, and PC3). The antiproliferative effect of the final compounds 3a-d and 7 on various cancer cell lines was determined by MTT assay and the results are shown in Table 2. Compound 3d exhibited the most potent inhibitory activity against all cancer cell lines, except MCF-7/Tam cancer cell lines and its $\mathrm{IC}_{50}$ values indicated sub-micromolar concentrations $(0.30-$ $0.76 \mu \mathrm{M})$, except MCF-7/Tam $(2.11 \mu \mathrm{M})$ cancer cell lines. Compound 3c showed the next potent inhibitory activity against cancer cell lines, and its $\mathrm{IC}_{50}$ values indicated submicromolar concentrations $(0.35-0.92 \mu \mathrm{M})$, except MCF-7/ Tam $(1.51 \mu \mathrm{M})$, and DU145 $(2.73 \mu \mathrm{M})$ cancer cell lines. Compound $\mathbf{3 b}$ also showed potent anticancer activities on

Table 1. Inhibition of HDAC activity by SAHA and compounds $\mathbf{3 a - 3 d}$ and $\mathbf{7}$

\begin{tabular}{ccccccc}
\hline \multirow{2}{*}{ HDAC inhibitors } & \multicolumn{5}{c}{ Compounds, $\mathrm{IC}_{50}(\mu \mathrm{M})$} \\
\cline { 2 - 7 } & SAHA & $\mathbf{3 a}$ & $\mathbf{3 b}$ & $\mathbf{3 c}$ & $\mathbf{3 d}$ & $\mathbf{7}$ \\
\hline $\begin{array}{c}\text { Total HDAC } \\
\text { (HeLa nuclear extract) }\end{array}$ & 5.882 & 2.903 & 0.428 & 0.336 & 0.214 & 10.145 \\
HDAC1 & 1.517 & 0.272 & 0.192 & 0.147 & 0.099 & 7.099 \\
HDAC2 & 2.574 & 2.457 & 1.987 & 2.015 & 0.105 & 5.784 \\
HDAC3 & 1.965 & 7.093 & 2.054 & 2.453 & 2.547 & 8.025 \\
HDAC4 & 10.311 & 31.570 & 16.781 & 12.228 & 41.086 & 53.487 \\
HDAC7 & 24.729 & 26.350 & 7.839 & 9.673 & 25.501 & 67.630 \\
\hline
\end{tabular}

Table 2. Effect of SAHA and compounds 3a-3d and $\mathbf{7}$ on cytotoxicity of human cancer cell lines

\begin{tabular}{llcccccc}
\hline \multirow{2}{*}{ Cell lines } & Origin & \multicolumn{5}{c}{ Compounds, $\mathrm{IC}_{50}(\mu \mathrm{M})$ at $48 \mathrm{hr}$} \\
\cline { 3 - 7 } & & SAHA & $\mathbf{3 a}$ & $\mathbf{3 b}$ & $\mathbf{3 c}$ & $\mathbf{3 d}$ & $\mathbf{7}$ \\
\hline MCF-7 & Breast & 4.21 & 5.48 & 1.08 & 0.92 & 0.54 & 12.54 \\
MDA-MB-231 & Breast & 5.78 & 6.29 & 2.43 & 0.87 & 0.76 & 10.37 \\
MCF-7/Dox & Breast & 3.14 & 4.25 & 1.37 & 0.56 & 0.42 & $>20$ \\
MCF-7/Tam & Breast & 5.24 & $>20$ & 2.58 & 1.51 & 2.11 & $>20$ \\
SK-OV-3 & Ovary & 2.25 & 3.12 & 1.25 & 0.58 & 0.42 & 6.67 \\
LNCaP & Prostate & 2.43 & 2.42 & 0.48 & 0.86 & 0.30 & $>20$ \\
DU145 & Prostate & 0.88 & 4.79 & 1.48 & 2.73 & 0.48 & $>20$ \\
PC3 & Prostate & 5.26 & 0.48 & 0.42 & 0.35 & 0.24 & 4.85 \\
\hline
\end{tabular}


prostate cancer cell lines including $\operatorname{LNCaP}(0.48 \mu \mathrm{M})$, and PC3 $(0.42 \mu \mathrm{M})$ cancer cell lines, and on the other cancer cells, $\mathrm{IC}_{50}$ values for anticancer activity were in range of 1.08-2.58 $\mu \mathrm{M}$. These three compounds, 3b, 3c and $\mathbf{3 d}$ exhibited 2 22-fold more potent anticancer activity than SAHA in all cancer cell lines, except DU145 cancer cell line. Compound 3a with a dimethylaminophenyl group as a cap showed more potent anticancer activity than SAHA only on PC3 cancer cell lines and exhibited similar anticancer activity to SAHA on the other cancer cell lines. Compound 7 which had a naphthalenyl ring as the cap moiety and a transposed amide structure showed similar anticancer activity to or less potent anticancer activity than SAHA, depending on the type of human cancer cell lines. It is notable that all the synthesized compounds 3a-d and 7 showed more potent anticancer activity than SAHA on PC3 cancer cell lines. These results show that $\mathbf{3 b}, \mathbf{3 c}$ and $\mathbf{3 d}$ may be a promise drug with a broad anticancer spectrum.

In summary, SAHA derivatives 3a-d and 7 with a bulky cap were synthesized in order to search for more potent anticancer agents with significant HDAC-inhibitory activity. EDC-mediated amide coupling reaction and a nucleophilic addition-elimination reaction with hydroxylamine were employed for the synthesis of the target compounds. Whereas compounds 3a and 7 showed a similar or a little less inhibitory effect to SAHA on total HDACs and sub-HDACs, compounds 3b, 3c and 3d showed significantly potent inhibitory activities on total HDACs (14 27-fold), HDAC1 (8 15-fold), HDAC2 (1.3 25-fold) and HDAC7 (1 3-fold), compared with SAHA. Although compound 7 showing the lowest HDAC inhibitory activity exhibited less potent anticancer activity than SAHA, compound 3a with a similar HDAC inhibitory activity to SAHA showed a similar anticancer activity to SAHA. Especially, compounds $\mathbf{3 b}, \mathbf{3 c}$ and 3d showing the highly potent HDAC inhibitory activity exhibited significantly potent anticancer activity (2 22-fold) against MCF-7, MDA-MB-231, MCF-7/Dox, MCF-7/Tam, SK-OV-3, LNCaP and PC3 human cancer cell lines, compared with SAHA. This study indicates proper bulkiness of the cap moiety of SAHA analogs may be closely correlated to high HDAC inhibition and anticancer activity.

\section{Experimental Section}

Melting points are uncorrected. NMR data were recorded on a $400 \mathrm{MHz}$ NMR spectrometer using tetramethylsilane (TMS) as an internal standard, and the chemical shifts are reported in ppm $(\delta)$. Coupling constants are reported in hertz. The abbreviations used are as follows: s (singlet), $d$ (doublet), m (multiplet), dd (doublet of doublet), br s (broad singlet) and quint (quintet). All the reactions described below were performed under argon or nitrogen atmosphere and monitored by thin layer chromatography (TLC). All anhydrous solvents were distilled over $\mathrm{CaH}_{2}$ or $\mathrm{Na}$ /benzophenone prior to use.

Methyl 8-(4-(Dimethylamino)phenylamino)-8-oxooctanoate (2a). To a stirred solution of $N^{1}, N^{1}$-dimethylbenzene- 1,4-diamine ( $80 \mathrm{mg}, 0.58 \mathrm{mmol}$ ), HOBt (1-hydroxybenzotriazole, $72 \mathrm{mg}, 0.53 \mathrm{mmol})$, and EDC $\cdot \mathrm{HCl}$ (1-ethyl-3-(3dimethylaminopropyl)carbodiimide hydrochloride, $153 \mathrm{mg}$, $0.80 \mathrm{mmol})$ in anhydrous methylene chloride $(2 \mathrm{~mL})$ was dropwise added suberic acid monoethyl ester (100 mg, 0.53 $\mathrm{mmol})$ at $0{ }^{\circ} \mathrm{C}$. After being stirred at room temperature for $48 \mathrm{~h}$, the reaction mixture was concentrated and the residue was diluted with ethyl acetate $(2 \times 50 \mathrm{~mL})$ and washed with $0.1 \mathrm{M} \mathrm{HCl}$ solution $(2 \times 25 \mathrm{~mL})$, and a saturated $\mathrm{NaHCO}_{3}$ solution $(25 \mathrm{~mL})$ subsequently. The organic layer was dried over $\mathrm{Na}_{2} \mathrm{SO}_{4}$ and filtered. The filtrate was concentrated and the residue was purified by silica gel column chromatography using methylene chloride and $\mathrm{MeOH}(45: 1)$ as the eluent to give amide $\mathbf{2 a}(106 \mathrm{mg}, 59 \%)$ as a yellowish solid; LRMS (FAB+) $m / z 307[\mathrm{M}+\mathrm{H}]^{+} ;{ }^{1} \mathrm{H}$ NMR $\left(500 \mathrm{MHz}, \mathrm{CDCl}_{3}\right)$ $\delta 7.34$ (d, $2 \mathrm{H}, J=9.0 \mathrm{~Hz}, \mathrm{Ph}), 7.30$ (br s, $1 \mathrm{H}, \mathrm{NH}), 6.68$ (br $\mathrm{s}, 2 \mathrm{H}, \mathrm{Ph}), 3.66\left(\mathrm{~s}, 3 \mathrm{H}, \mathrm{OCH}_{3}\right), 2.91\left(\mathrm{~s}, 6 \mathrm{H}, 2 \times \mathrm{NCH}_{3}\right), 2.30$ (m, $4 \mathrm{H}, 2-\mathrm{CH}_{2}, 7-\mathrm{CH}_{2}$ ), 1.71 (quint, $2 \mathrm{H}, J=7.5 \mathrm{~Hz}, 6-\mathrm{CH}_{2}$ ), 1.62 (quint, $2 \mathrm{H}, J=7.5 \mathrm{~Hz}, 3-\mathrm{CH}_{2}$ ), 1.40-1.33 (m, $4 \mathrm{H}, 4-$ $\left.\mathrm{CH}_{2}, 5-\mathrm{CH}_{2}\right) ;{ }^{13} \mathrm{C} \mathrm{NMR}\left(100 \mathrm{MHz}, \mathrm{CDCl}_{3}\right) \delta 174.51$ $\mathrm{C}=\mathrm{O}), 171.36(8-\mathrm{C}=\mathrm{O}), 148.14(\mathrm{Ph}), 128.33(\mathrm{Ph}), 122.01$ $(\mathrm{Ph}), 113.40(\mathrm{Ph}), 51.71\left(\mathrm{O}-\mathrm{CH}_{3}\right), 41.24\left(\mathrm{NCH}_{3}\right), 37.57$, 34.18, 29.04, 29.00, 25.74, 24.95.

Methyl 8-Oxo-8-(4-phenoxyphenylamino)octanoate (2b). 4-Phenoxyphenyl analog $\mathbf{2 b}$ was synthesized by a procedure similar to that described for 2a: yield 62\%; LRMS (FAB+) $m / z 356[\mathrm{M}+\mathrm{H}]^{+} ;{ }^{1} \mathrm{H} \mathrm{NMR}\left(400 \mathrm{MHz}, \mathrm{CDCl}_{3}\right) \delta 7.98(\mathrm{~s}, 1$ H, NH), 7.47 (d, 2 H, J=8.8 Hz, Ph), 7.27 (t, $2 \mathrm{H}, J=7.6$ $\mathrm{Hz}, \mathrm{Ph}), 7.04$ (t, $1 \mathrm{H}, J=7.2 \mathrm{~Hz}, \mathrm{Ph}), 6.93$ (d, $2 \mathrm{H}, J=7.2$ $\mathrm{Hz}, \mathrm{Ph}), 6.91$ (d, $2 \mathrm{H}, J=8.8 \mathrm{~Hz}, \mathrm{Ph}), 3.63$ (s, $3 \mathrm{H}, \mathrm{OCH}_{3}$ ), 2.30 (t, $\left.2 \mathrm{H}, J=8.0 \mathrm{~Hz}, 2-\mathrm{CH}_{2}\right), 2.27$ (t, $2 \mathrm{H}, J=7.6 \mathrm{~Hz}, 7-$ $\mathrm{CH}_{2}$ ), 1.68 (quint, $2 \mathrm{H}, J=6.4 \mathrm{~Hz}, 6-\mathrm{CH}_{2}$ ), 1.59 (quint, $2 \mathrm{H}$, $\left.J=7.2 \mathrm{~Hz}, 3-\mathrm{CH}_{2}\right), 1.32\left(\mathrm{~m}, 4 \mathrm{H}, 4-\mathrm{CH}_{2}, 5-\mathrm{CH}_{2}\right) ;{ }^{13} \mathrm{C} \mathrm{NMR}$ $\left(100 \mathrm{MHz}, \mathrm{CDCl}_{3}\right) \delta 174.58(1-\mathrm{C}=\mathrm{O}), 171.92(8-\mathrm{C}=\mathrm{O})$, $157.77(\mathrm{Ph}), 153.44(\mathrm{Ph}), 133.96(\mathrm{Ph}), 129.94(\mathrm{Ph}), 123.25$ $(\mathrm{Ph}), 121.94(\mathrm{Ph}), 119.74(\mathrm{Ph}), 118.55(\mathrm{Ph}), 51.76\left(\mathrm{O}-\mathrm{CH}_{3}\right)$, 37.52, 34.16, 29.03, 28.97, 25.65, 24.91.

Methyl 8-Oxo-8-(4-phenylamino)phenylamino)octanoate (2c). 4-Phenylaminophenyl analog 2c was synthesized by a procedure similar to that described for 2a: yield 49\%; LRMS $(\mathrm{FAB}+) \mathrm{m} / \mathrm{z} 355[\mathrm{M}+\mathrm{H}]^{+} ;{ }^{1} \mathrm{H}$ NMR $\left(400 \mathrm{MHz}, \mathrm{CDCl}_{3}\right) \delta$ 7.56 (s, $1 \mathrm{H}, \mathrm{CONH}), 7.38$ (d, $2 \mathrm{H}, J=8.8 \mathrm{~Hz}, \mathrm{Ph}), 7.21$ (t, 2 $\mathrm{H}, J=8.4 \mathrm{~Hz}, \mathrm{Ph}), 7.00(\mathrm{~d}, 2 \mathrm{H}, J=8.8 \mathrm{~Hz}, \mathrm{Ph}), 6.98(\mathrm{~d}, 2 \mathrm{H}$, $J=8.8 \mathrm{~Hz}, \mathrm{Ph}), 6.86(\mathrm{t}, 1 \mathrm{H}, J=7.2 \mathrm{~Hz}, \mathrm{Ph}), 5.76(\mathrm{~s}, 1 \mathrm{H}$, $\mathrm{NH}), 3.64\left(\mathrm{~s}, 3 \mathrm{H}, \mathrm{OCH}_{3}\right), 2.30\left(\mathrm{t}, 2 \mathrm{H}, J=7.2 \mathrm{~Hz}, 2-\mathrm{CH}_{2}\right)$, 2.27 (t, $2 \mathrm{H}, J=7.2 \mathrm{~Hz}, 7-\mathrm{CH}_{2}$ ), 1.69 (quint, $2 \mathrm{H}, J=6.8 \mathrm{~Hz}$, $3-\mathrm{CH}_{2}$ ), 1.60 (quint, $\left.2 \mathrm{H}, J=6.8 \mathrm{~Hz}, 6-\mathrm{CH}_{2}\right), 1.33(\mathrm{~m}, 4 \mathrm{H}$, $\left.4-\mathrm{CH}_{2}, 5-\mathrm{CH}_{2}\right) ;{ }^{13} \mathrm{C} \mathrm{NMR}\left(100 \mathrm{MHz}, \mathrm{CDCl}_{3}\right) \delta 174.59$ (1$\mathrm{C}=\mathrm{O}), 171.60(8-\mathrm{C}=\mathrm{O}), 143.84(\mathrm{Ph}), 139.64(\mathrm{Ph}), 131.94$ $(\mathrm{Ph}), 129.55(\mathrm{Ph}), 121.77(\mathrm{Ph}), 120.71(\mathrm{Ph}), 119.16(\mathrm{Ph})$, $117.20(\mathrm{Ph}), 51.76\left(\mathrm{OCH}_{3}\right), 37.59,34.18,29.03,28.98$, 25.69, 24.93.

Methyl 8-(9H-Fluoren-2-ylamino)-8-oxooctanoate (2d). Fluorenyl analog 2d was synthesized by a procedure similar to that described for $\mathbf{2 a}$ : yield $72 \%$; ${ }^{1} \mathrm{H}$ NMR $(400 \mathrm{MHz}$, $\left.\mathrm{CDCl}_{3}\right) \delta 7.88(\mathrm{~s}, 1 \mathrm{H}, \mathrm{Ar}), 7.66(\mathrm{~m}, 2 \mathrm{H}, \mathrm{Ar}), 7.49(\mathrm{~m}, 1 \mathrm{H}$, Ar), 7.34 (m, 2 H, Ar), 7.24 (t, $1 \mathrm{H}, J=6.8 \mathrm{~Hz}, \mathrm{Ar}), 3.82$ (s, 
2H, benzylic $\mathrm{H}), 3.65\left(\mathrm{~s}, 3 \mathrm{H}, \mathrm{OCH}_{3}\right), 2.34(\mathrm{t}, 2 \mathrm{H}, J=6.8$ $\mathrm{Hz}, 2-\mathrm{CH}_{2}$ ), 2.29 (t, $2 \mathrm{H}, J=6.8 \mathrm{~Hz}, 7-\mathrm{CH}_{2}$ ), 1.73 (quint, 2 $\mathrm{H}, J=6.0 \mathrm{~Hz}, 3-\mathrm{CH}_{2}$ ), 1.62 (quint, $2 \mathrm{H}, J=6.8 \mathrm{~Hz}, 6-\mathrm{CH}_{2}$ ), $1.36\left(\mathrm{~m}, 4 \mathrm{H}, 4-\mathrm{CH}_{2}, 5-\mathrm{CH}_{2}\right) ;{ }^{13} \mathrm{C} \mathrm{NMR}\left(100 \mathrm{MHz}, \mathrm{CDCl}_{3}\right)$ $\delta 174.55(1-\mathrm{C}=\mathrm{O}), 171.72(8-\mathrm{C}=\mathrm{O}), 144.50(\mathrm{Ar}), 143.38$ (Ar), 141.54 (Ar), 138.14 (Ar), 137.11 (Ar), 126.97 (Ar), 126.49 (Ar), 125.19 (Ar), 120.27 (Ar), 119.70 (Ar), 118.83 (Ar), 117.12 (Ar), $51.74\left(\mathrm{OCH}_{3}\right), 37.81,37.22$, 34.16, 28.96, 28.98, 25.61, 24.90.

$N^{\mathbf{1}}$-(4-(Dimethylamino)phenyl)- $\boldsymbol{N}^{\mathbf{8}}$-hydroxyoctanediamide (3a). To a stirred solution of $\mathbf{2 a}(106 \mathrm{mg}, 0.34 \mathrm{mmol})$ in $\mathrm{MeOH}(4 \mathrm{~mL})$ was added $50 \%$ hydroxylamine aqueous solution $(2.2 \mathrm{~mL})$ at $0{ }^{\circ} \mathrm{C}$. The reaction mixture was stirred at room temperature for $2 \mathrm{~d}$. In succession, to this reaction mixture was added $50 \%$ hydroxylamine aqueous solution $(0.5 \mathrm{~mL})$ and the reaction mixture was stirred at room temperature for an additional $3 \mathrm{~d}$. The precipitated solid was filtered, washed with water, air-dried, and dried under high vacuum to give hydroxamic acid $\mathbf{3 a}$ as a white solid $(61 \mathrm{mg}$, 57\%); mp 155.7-160 ${ }^{\circ} \mathrm{C}$; LRMS (FAB+) $m / z 308[\mathrm{M}+\mathrm{H}]^{+}$; ${ }^{1} \mathrm{H}$ NMR (400 MHz, CD $\left.3 \mathrm{OD}\right) \delta 7.32(\mathrm{~d}, 2 \mathrm{H}, J=8.8 \mathrm{~Hz}$, $\mathrm{Ph}), 6.73(\mathrm{~d}, 2 \mathrm{H}, J=8.8 \mathrm{~Hz}, \mathrm{Ph}), 2.86\left(\mathrm{~s}, 6 \mathrm{H}, 2 \times \mathrm{NCH}_{3}\right)$, 2.30 (t, $\left.2 \mathrm{H}, J=7.2 \mathrm{~Hz}, 2-\mathrm{CH}_{2}\right), 2.07$ (t, $2 \mathrm{H}, J=7.2 \mathrm{~Hz}, 7-$ $\left.\mathrm{CH}_{2}\right), 1.68-1.59$ (m, $\left.4 \mathrm{H}, 3-\mathrm{CH}_{2}, 6-\mathrm{CH}_{2}\right), 1.39-1.33$ (m, $4 \mathrm{H}$, $\left.4-\mathrm{CH}_{2}, 5-\mathrm{CH}_{2}\right) ;{ }^{13} \mathrm{C}$ NMR $\left(100 \mathrm{MHz}, \mathrm{CD}_{3} \mathrm{OD}\right) \delta 173.05(8-$ $\mathrm{C}=\mathrm{O}), 171.76(1-\mathrm{C}=\mathrm{O}), 148.33(\mathrm{Ph}), 128.78(\mathrm{Ph}), 121.79$ $(\mathrm{Ph}), 113.29(\mathrm{Ph}), 40.20\left(\mathrm{NCH}_{3}\right), 36.48,32.48,28.70,28.62$, 25.68, 25.40.

$N^{1}$-Hydroxy- $N^{\mathbf{8}}$-(4-phenoxyphenyl)octanediamide (3b). 4-Phenoxyphenyl analog $\mathbf{3 b}$ was synthesized by a procedure similar to that described for 3a: yield 67\%; mp 162.5-163.1 ${ }^{\circ} \mathrm{C}$; LRMS (FAB+) $m / z 357[\mathrm{M}+\mathrm{H}]^{+}$; ${ }^{1} \mathrm{H} \mathrm{NMR}(400 \mathrm{MHz}$, DMSO- $\left.d_{6}\right) \delta 10.32(\mathrm{~s}, 1 \mathrm{H}, \mathrm{NHOH}), 9.86(\mathrm{~s}, 1 \mathrm{H}, \mathrm{NH}), 8.65$ (s, 1 H, OH), 7.57 (d, $2 \mathrm{H}, J=8.8 \mathrm{~Hz}, \mathrm{Ph}), 7.32$ (t, 2H, $J=$ $7.6 \mathrm{~Hz}, \mathrm{Ph}), 7.05$ (t, $1 \mathrm{H}, J=7.2 \mathrm{~Hz}, \mathrm{Ph}), 6.93$ (d, $2 \mathrm{H}, J=$ $8.4 \mathrm{~Hz}, \mathrm{Ph}), 6.91(\mathrm{~d}, 2 \mathrm{H}, J=8.8 \mathrm{~Hz}, \mathrm{Ph}), 2.24(\mathrm{t}, 2 \mathrm{H}, J=$ $7.2 \mathrm{~Hz}, 7-\mathrm{CH}_{2}$ ), 1.90 (t, $2 \mathrm{H}, J=7.6 \mathrm{~Hz}, 2-\mathrm{CH}_{2}$ ), 1.54 (quint, $2 \mathrm{H}, J=6.4 \mathrm{~Hz}, 6-\mathrm{CH}_{2}$ ), 1.45 (quint, $2 \mathrm{H}, J=6.4 \mathrm{~Hz}, 3-\mathrm{CH}_{2}$ ), 1.28-1.20 (m, $\left.4 \mathrm{H}, 4-\mathrm{CH}_{2}, 5-\mathrm{CH}_{2}\right) ;{ }^{13} \mathrm{C} \mathrm{NMR}(100 \mathrm{MHz}$, DMSO- $\left.d_{6}\right) \delta 171.70(8-\mathrm{C}=\mathrm{O}), 169.74(1-\mathrm{C}=\mathrm{O}), 158.13(\mathrm{Ph})$, $152.03(\mathrm{Ph}), 135.99(\mathrm{Ph}), 130.60(\mathrm{Ph}), 123.54(\mathrm{Ph}), 121.33$ $(\mathrm{Ph}), 120.15(\mathrm{Ph}), 118.39(\mathrm{Ph}), 36.96,32.89,29.08,25.73$, 25.70 .

\section{$N^{\mathbf{1}}$-Hydroxy- $\boldsymbol{N}^{\mathbf{8}}$-(4-(phenylamino)phenyl)octanediamide} (3c). 4-Phenylaminophenyl analog $3 \mathbf{c}$ was synthesized by a procedure similar to that described for $3 \mathrm{a}$ : yield $65 \%$; mp 155.2-155.8 ${ }^{\circ} \mathrm{C}$; LRMS (FAB+) $m / z 356[\mathrm{M}+\mathrm{H}]^{+} ;{ }^{1} \mathrm{H}$ NMR $\left(500 \mathrm{MHz}, \mathrm{DMSO}-d_{6}\right) \delta 10.34(\mathrm{~s}, 1 \mathrm{H}, \mathrm{NHOH}), 9.69$ (s, $1 \mathrm{H}$, $\mathrm{NH}), 8.67$ (s, 1H, anilinic NH), 7.99 (s, 1H, OH), 7.45 (d, $2 \mathrm{H}, J=9.0 \mathrm{~Hz}, \mathrm{Ph}), 7.18$ (t, 2H, $J=7.5 \mathrm{~Hz}, \mathrm{Ph}), 7.00$ (d, 2H, $J=9.0 \mathrm{~Hz}, \mathrm{Ph}), 6.98$ (d, $2 \mathrm{H}, J=8.5 \mathrm{~Hz}, \mathrm{Ph}), 6.74$ (t, $1 \mathrm{H}, J$ $=7.5 \mathrm{~Hz}, \mathrm{Ph}), 2.24\left(\mathrm{t}, 2 \mathrm{H}, J=7.0 \mathrm{~Hz}, 7-\mathrm{CH}_{2}\right), 1.93(\mathrm{t}, 2 \mathrm{H}, J$ $=7.5 \mathrm{~Hz}, 2-\mathrm{CH}_{2}$ ), 1.56 (quint, $2 \mathrm{H}, J=6.5 \mathrm{~Hz}, 6-\mathrm{CH}_{2}$ ), 1.48 (quint, $\left.2 \mathrm{H}, J=7.0 \mathrm{~Hz}, 3-\mathrm{CH}_{2}\right), 1.33-1.25\left(\mathrm{~m}, 4 \mathrm{H}, 4-\mathrm{CH}_{2}, 5-\right.$ $\left.\mathrm{CH}_{2}\right) ;{ }^{13} \mathrm{C}$ NMR $\left(100 \mathrm{MHz}, \mathrm{DMSO}-d_{6}\right) \delta 171.27(8-\mathrm{C}=\mathrm{O})$, $169.76(1-\mathrm{C}=\mathrm{O}), 144.82(\mathrm{Ph}), 139.15(\mathrm{Ph}), 133.01(\mathrm{Ph})$, $129.79(\mathrm{Ph}), 121.01(\mathrm{Ph}), 119.52(\mathrm{Ph}), 118.57(\mathrm{Ph}), 116.28$
(Ph), 44.54, 36.92, 32.90, 29.08, 25.83, 25.72.

$\boldsymbol{N}^{\mathbf{1}}$-(9H-Fluoren-2-yl)- $\boldsymbol{N}^{\mathbf{8}}$-hydroxyoctanediamide (3d). Fluorenyl analog $\mathbf{3 d}$ was synthesized by a procedure similar to that described for 3a: yield $57 \%$; mp $170.5-170.8{ }^{\circ} \mathrm{C} ;{ }^{1} \mathrm{H}$ NMR (500 MHz, DMSO-d $) \delta 10.36$ (s, $1 \mathrm{H}, \mathrm{NHOH}), 9.95$ (s, $1 \mathrm{H}, \mathrm{NH}), 8.70$ (s, $1 \mathrm{H}, \mathrm{OH}), 7.94$ (s, $1 \mathrm{H}, \mathrm{Ar}), 7.77$ (d, 2 $\mathrm{H}, J=7.5 \mathrm{~Hz}, \mathrm{Ar}), 7.53$ (d, $1 \mathrm{H}, J=8.0 \mathrm{~Hz}, \mathrm{Ar}), 7.51(\mathrm{~d}, 1 \mathrm{H}$, $J=7.5 \mathrm{~Hz}, \mathrm{Ar}), 7.32$ (t, $1 \mathrm{H}, J=7.5 \mathrm{~Hz}, \mathrm{Ar}), 7.23$ (t, $1 \mathrm{H}, J=$ $7.0 \mathrm{~Hz}, \mathrm{Ar}), 2.31$ (t, $\left.2 \mathrm{H}, J=7.0 \mathrm{~Hz}, 2-\mathrm{CH}_{2}\right), 1.94$ (t, $2 \mathrm{H}, J=$ $7.0 \mathrm{~Hz}, 7-\mathrm{CH}_{2}$ ), 1.59 (quint, $\left.2 \mathrm{H}, J=6.0 \mathrm{~Hz}, 3-\mathrm{CH}_{2}\right), 1.49$ (quint, $\left.2 \mathrm{H}, J=6.5 \mathrm{~Hz}, 6-\mathrm{CH}_{2}\right), 1.28\left(\mathrm{~m}, 4 \mathrm{H}, 4-\mathrm{CH}_{2}, 5-\right.$ $\left.\mathrm{CH}_{2}\right) ;{ }^{13} \mathrm{C}$ NMR $\left(100 \mathrm{MHz}, \mathrm{DMSO}-d_{6}\right) \delta 171.89(1-\mathrm{C}=\mathrm{O})$, $169.85(8-\mathrm{C}=\mathrm{O}), 144.36$ (Ar), $143.40(\mathrm{Ar}), 141.71$ (Ar), 139.08 (Ar), 136.76 (Ar), 127.36 (Ar), 126.66 (Ar), 125.64 (Ar), 120.70 (Ar), 120.03 (Ar), 118.53 (Ar), 116.56 (Ar), 37.15, 32.93, 29.10, 25.79, 25.73.

Methyl 6-(2-(Naphthalen-1-yl)acetamido)hexanoate (6). To a stirred solution of 2-(naphthalen-1-yl)acetic acid (200 $\mathrm{mg}, 1.07 \mathrm{mmol})$ in 1,4-dioxane $(2 \mathrm{~mL})$ were added $\mathrm{HOBt}$ (363 mg, $2.68 \mathrm{mmol})$ and $\mathrm{EDC} \cdot \mathrm{HCl}(309 \mathrm{mg}, 1.61 \mathrm{mmol})$ successively at room temperature with stirring. This reaction mixture was stirred constantly at the same temperature for $2 \mathrm{~h}$ and the volatiles were evaporated under reduced pressure. The concentrated residue was diluted with ethyl acetate $(50 \mathrm{~mL})$ and washed with $\mathrm{NaHCO}_{3}(25 \mathrm{~mL})$. The organic layer was dried over anhydrous $\mathrm{MgSO}_{4}$, filtered, and evaporated under reduced pressure to give compound 5 (310 $\mathrm{mg}, 95 \%$ ) as a sticky yellowish oil. To a stirred solution of $\mathbf{5}$ (310 mg, $1.02 \mathrm{mmol})$ in 1,4-dioxane $(2 \mathrm{~mL})$ were added methyl 6-aminohexanoate hydrochloride (186 mg, 1.02 mmol) and $N$-methylmorpholine $(0.19 \mathrm{~mL}, 2.04 \mathrm{mmol})$ successively at room temperature with stirring. After the reaction mixture was stirred constantly at the same temperature for $2 \mathrm{~d}$, the resulting solution was filtered off through a pad of Celite and washed with methylene chloride and $\mathrm{MeOH}$ (70:1). The filtrate was concentrated and was purified by silica gel column chromatography using methylene chloride and $\mathrm{MeOH}(70: 1)$ as the eluent to give amide $\mathbf{6}$ (126 $\mathrm{mg}, 37 \%$ from 4) as a yellow oil; LRMS (FAB+) $m / z 314$ $[\mathrm{M}+\mathrm{H}]^{+} ;{ }^{1} \mathrm{H} \mathrm{NMR}\left(400 \mathrm{MHz}, \mathrm{CDCl}_{3}\right) \delta$ 7.93-7.90 (m, $1 \mathrm{H}$, $\mathrm{Ph})$, 7.85-7.83 (m, 1 H, Ar), 7.78 (d, $1 \mathrm{H}, J=7.2 \mathrm{~Hz}, \mathrm{Ar})$, 7.52-7.45 (m, 2 H, Ar), 7.41 (t, $1 \mathrm{H}, J=7.6 \mathrm{~Hz}, \mathrm{Ar}), 7.35$ (d, $1 \mathrm{H}, J=7.2 \mathrm{~Hz}, \mathrm{Ar}$ ), 5.42 (br s, $1 \mathrm{H}, \mathrm{NH}), 3.96$ (s, $2 \mathrm{H}$, $\mathrm{CH}_{2} \mathrm{Ar}$ ), 3.59 (s, $3 \mathrm{H}, \mathrm{OCH}_{3}$ ), 3.08 (q, $2 \mathrm{H}, J=6.8 \mathrm{~Hz}, 6-$ $\mathrm{CH}_{2}$ ), 2.12 (t, $2 \mathrm{H}, J=7.6 \mathrm{~Hz}, 2-\mathrm{CH}_{2}$ ), 1.43 (quint, $2 \mathrm{H}, J=$ $8.0 \mathrm{~Hz}, 3-\mathrm{CH}_{2}$ ), 1.26 (quint, $2 \mathrm{H}, J=7.2 \mathrm{~Hz}, 5-\mathrm{CH}_{2}$ ), 1.07$0.98\left(\mathrm{~m}, 2 \mathrm{H}, 4-\mathrm{CH}_{2}\right) ;{ }^{13} \mathrm{C} \mathrm{NMR}\left(100 \mathrm{MHz}, \mathrm{CDCl}_{3}\right) \delta 174.15$ $(1-\mathrm{C}=\mathrm{O}), 171.05(\mathrm{NHC}=\mathrm{O}), 134.14(\mathrm{Ar}), 132.25(\mathrm{Ar}), 131.46$ (Ar), 128.98 (Ar), 128.66 (Ar), 128.55 (Ar), 126.93 (Ar), 126.40 (Ar), 125.85 (Ar), $124.04(\mathrm{Ar}), 51.69\left(\mathrm{OCH}_{3}\right), 42.04$, 39.49, 33.97, 29.20, 26.31, 24.56.

$\mathrm{N}$-Hydroxy-6-(2-(naphthalen-1-yl)acetamido)hexanamide (7). Naphthalenyl analog 7 was synthesized by a procedure similar to that described for 3a: yield 51\%; mp 135.2-135.9 ${ }^{\circ} \mathrm{C}$; LRMS (FAB+) $m / z 315[\mathrm{M}+\mathrm{H}]^{+}$; ${ }^{1} \mathrm{H}$ NMR $(500 \mathrm{MHz}$, $\left.\mathrm{CD}_{3} \mathrm{OD}\right) \delta 8.03$ (d, $\left.1 \mathrm{H}, J=8.5 \mathrm{~Hz}, \mathrm{Ar}\right), 7.87$ (d, $1 \mathrm{H}, J=8.0$ $\mathrm{Hz}, \mathrm{Ar}), 7.79$ (d, $1 \mathrm{H}, J=7.5 \mathrm{~Hz}, \mathrm{Ar}), 7.53$ (t, $1 \mathrm{H}, J=7.5$ 
$\mathrm{Hz}, \mathrm{Ar}), 7.49$ (t, $1 \mathrm{H}, J=7.5 \mathrm{~Hz}, \mathrm{Ar}), 7.44(\mathrm{t}, 1 \mathrm{H}, J=7.0 \mathrm{~Hz}$, $\mathrm{Ar}), 7.41(\mathrm{~d}, 1 \mathrm{H}, J=7.5 \mathrm{~Hz}, \mathrm{Ar}), 3.97\left(\mathrm{~s}, 2 \mathrm{H}, \mathrm{CH}_{2} \mathrm{Ar}\right), 3.16$ (t, $\left.2 \mathrm{H}, J=7.0 \mathrm{~Hz}, \mathrm{NCH}_{2}\right), 2.03\left(\mathrm{t}, 2 \mathrm{H}, J=7.5 \mathrm{~Hz}, 2-\mathrm{CH}_{2}\right.$ ), 1.56 (quint, $2 \mathrm{H}, J=7.5 \mathrm{~Hz}, 3-\mathrm{CH}_{2}$ ), 1.47 (quint, $2 \mathrm{H}, J=7.5$ $\left.\mathrm{Hz}, 5-\mathrm{CH}_{2}\right), 1.29-1.24\left(\mathrm{~m}, 2 \mathrm{H}, 4-\mathrm{CH}_{2}\right) ;{ }^{13} \mathrm{C}$ NMR $(100$ $\left.\mathrm{MHz}, \mathrm{CDCl}_{3}\right) \delta 172.75(\mathrm{NHC}=\mathrm{O}), 171.64(1-\mathrm{C}=\mathrm{O}), 134.19$ (Ar), 132.39 (Ar), $131.84(\mathrm{Ar}), 128.53(\mathrm{Ar}), 127.88(\mathrm{Ar})$, 127.70 (Ar), 126.07 (Ar), 125.63 (Ar), 125.43 (Ar), 123.69 (Ar), 40.32, 39.20, 32.41, 28.78, 26.15, 25.15.

General for Cell Culture, Cell Viability Test and Assay of HDAC Enzyme Activity. SAHA and all chemicals used for bioassay were purchased from Sigma-Aldrich (St. Louis, MO, USA). SAHA and test compounds 3a-3d and 7 were dissolved in sterile dimethyl sulfoxide (DMSO) to generate a $10 \mathrm{mM}$ stock solution. The solution was stored at $-80^{\circ} \mathrm{C}$. Subsequent dilutions were made in RPMI-1640 (Gibco, Rockville, MD, USA).

Cell Lines and Culture. The cell lines, MCF-7, MDAMB-231, PC3, DU-145, LNCaP, and SK-OV-3 cells were purchased from the American Type Culture Collection (ATCC) (Manassas, VA, USA). All cells were maintained as monolayers at $37^{\circ} \mathrm{C}$ in an atmosphere containing $5 \% \mathrm{CO}_{2} /$ air in RPMI-1640 (Gibco, Rockville, MD) containing $2 \mathrm{mM}$ L-glutamate, $10 \%$ heat-inactivated fetal bovine serum (FBS, Gibco), $1.25 \mathrm{mM}$ HEPES (Gibco) and $100 \mathrm{U} / \mathrm{mL}$ penicillin/ streptomycin (Gibco).

Cell Viability Test. Cytotoxicity was assessed by 3-(4,5dimethylthiazole-2-yl)-2,5-diphenyltetrazolium bromide (MTT) assay. For the compound treatment, the cells were plated for $48 \mathrm{~h}$ in RPMI-1640 containing 10\% FBS. The medium was then changed to RPMI-1640 containing 5\% charcoal-dextran treated FBS (CD-FBS) with various concentrations of test compounds, SAHA, 3a-3d and 7. The cultures were initiated in 96-well microtiter plates at a density of $1 \times 10^{4}$ cells per well. Cells were allowed to attach for $48 \mathrm{~h}$ before treatment with SAHA, 3a-3d and 7. At the end of the treatment period, $15 \mu \mathrm{L}$ of the MTT reagent $(5$ $\mathrm{mg} / \mathrm{mL}$ ) was added to each well. After $4 \mathrm{~h}$ incubation at $37^{\circ} \mathrm{C}$, the supernatant was aspirated, and formazan crystals were dissolved in $100 \mu \mathrm{L}$ DMSO at $37^{\circ} \mathrm{C}$ for 10 min with gentle agitation. The absorbance per well was measured at $540 \mathrm{~nm}$ with a VERS Amax Microplate Reader (Molecular Devices Corp.). Assay was done in triplicate. The $\mathrm{IC}_{50}$ values were then determined for each compounds from a plot of log (compound concentration) versus percentage of cell killed.

Assay of HDAC Enzyme Activity. The HDAC enzymes were purchased from BPS Bioscience (San Diego, CA, USA) and the HDAC enzyme assay was performed using a Fluorogenic HDAC Assay Kit (BPS Bioscience) according to the manufacturer's instructions. Briefly, HDAC enzymes were incubated with vehicle or various concentrations of SAHA, 3a-3d and 7 for $30 \mathrm{~min}$ at $37^{\circ} \mathrm{C}$ in the presence of an HDAC fluorimetric substrate. The HDAC assay developer (which produces a fluorophore in the reaction mixture) was added, and the fluorescence was measured using VICTOR 3 (PerkinElmer, Waltham, MA, USA) with excitation at 360 $\mathrm{nm}$ and emission at $460 \mathrm{~nm}$. The measured activities were substracted by calculated using GraphPad Prism (Graphpad Software, San Diego, CA, USA).

Statistical Analysis. All the data is represented as the average of the values obtained $\pm \mathrm{SD}$. A paired Student's $t$ test was used to determine the statistical significance. A $p$ value $<0.05$, or $p$-value $<0.01$ was considered statistically significant.

Acknowledgments. This work was supported by the Basic Science Research Program through the National Research Foundation of Korea (NRF) funded by the Ministry of Education, Science and Technology (No. 2010-0012038).

\section{References}

1. Turner, B. M. Nat. Cell. Biol. 2000, 22, 836 .

2. Jenuwein, T.; Allis, C. D. Science 2001, 293, 1074.

3. Davie, J. R. Curr. Opin. Genet. Rev. 1998, 8, 173.

4. Finnin, M. S.; Donigian, J. R.; Cohen, A.; Richon, V. M.; Rifkind, R. A.; Marks, P. A.; Breslow, R.; Pavletich, N. P. Nature 1999, $401,188$.

5. Sakimura, R.; Tanaka, K.; Nakatani, F.; Matsunobu, T.; Hanada, M.; Okada, T.; Nakamura, T.; Matsumoto, Y.; Iwamoto, Y. Int. J. Cancer 2005, 116, 784

6. Bolden, J. E.; Peart, M. J.; Johnstone, R. W. Nat. Rev. Drug Discov. 2006, 5, 769.

7. Kuo, P. H.; Carlson, K. R.; Christensen, I.; Girardi, M.; Heald, P. W. Mol. Imaging Biol. 2008, 10, 306.

8. Ueda, T.; Takai, N.; Nishida, M.; Nasu, K.; Narahara, H. Int. J. Mol. Med. 2007, 19, 301.

9. Yoshida, M.; Kijima, M.; Akita, M.; Beppu, T. J. Biol. Chem. 1990, 265, 17174

10. Pina, I. C.; Gautschi, J. T.; Wang, G.-Y.-S.; Sanders, M. L.; Schmitz, F. J.; France, D.; Cornell-Kennon, S.; Sambucetti, L. C.; Remiszewski, S. W.; Perez, L. B.; Bair, K. W.; Crews, P. J. Org. Chem. 2003, 68, 3866.

11. Kijima, M.; Yoshida, M.; Sugita, K.; Horinouchi, S.; Beppu, T. J. Biol. Chem. 1993, 268, 22429.

12. Butler, L. M.; Agus, D. B.; Scher, H. I.; Higgins, B.; Rose, A.; Cordon-Cardo, C.; Thaler, H. T.; Rifkind, R. A.; Marks, P. A.; Richon, V. M. Cancer Res. 2000, 60, 5165.

13. Vigushin, D. M.; Ali, S.; Pace, P. E.; Mirsaidi, N.; Ito, K.; Adcock, I.; Coombes, R. C. Clin. Cancer Res. 2001, 7, 971.

14. Breslow, R.; Jursic, B.; Yan, Z. F.; Friedman, E.; Leng, L.; Ngo, L.; Rifkind, R. A.; Marks, P. A. Proc. Natl. Acad. Sci. USA 1991, $88,5542$.

15. Yamamoto, S.; Tanaka, K.; Sakimura, R.; Okada, T.; Nakamura, T.; Li, Y.; Takasaki, M.; Nakabeppu, Y.; Iwamoto, Y. Anticancer Res. 2008, 28, 1585.

16. Ruefli, A. A.; Ausserlechner, M. J.; Bernhard, D.; Sutton, V. R.; Tainton, K. M.; Kofler, R.; Smyth, M. J.; Johnstone, R. W. Proc. Natl. Acad. Sci. USA 2001, 98, 10833.

17. Mai, A.; Massa, S.; Rotili, D.; Cerbara, I.; Valente, S.; Pezzi, R.; Simeoni, S.; Ragno, R. Medicinal Res. Rev. 2005, 25, 261.

18. Gros, L.; Lorente, S. O.; Jimenez, C. J.; Yardley, V.; Rattray, L.; Wharton, H.; Little, S.; Croft, S. L.; Ruiz-Perez, L. M.; GonzalezPacanowska, D.; Gilbert, I. H. J. Med. Chem. 2006, 49, 6094.

19. Jones, J. In Amino Acid and Peptide Synthesis, $2^{\text {nd }}$ ed.; Oxford University Press: New York, USA, 2002; p 32. 